\title{
Conhecimento e práticas dos agentes comunitários de saúde sobre hanseníase em um município hiperendêmico
}

\author{
Knowledge and practices of community health \\ agents on leprosy in a hyperendemic municipality
}

Camila Martins de Oliveira Universidade Estadual Vale do Acaraú, Sobral/ CE - Brasil MARIA Socorro Carneiro LINHARES

Universidade Estadual Vale do Acaraú, Sobral/ CE - Brasil Francisco Rosemiro GuimaRÃes Ximenes Neto Universidade Estadual Vale do Acaraú, Sobral/ CE - Brasil Irisdalva Maria Vieira Pessoa Mendes

Secretaria de Saúde de Sobral, Sobral/ CE - Brasil

Lígia Regina Franco Sansigolo KERR

Universidade Federal Do Ceará (UFC), Fortaleza/ CE - Brasil

\section{Resumo}

Objetivo: Descrever o conhecimento dos agentes comunitários de saúde (ACS) sobre hanseníase, suas práticas na atenção aos casos de sua microárea e identificar os tipos de qualificações recebidas acerca da doença.

Materiais e Métodos: Estudo exploratório-descritivo, sob abordagem qualitativa e quantitativa. Os sujeitos do estudo foram os ACS que atuam em três territórios da Estratégia Saúde da Família (ESF) do município de Sobral, Ceará, com maior número de casos de hanseníase registrados. As informações foram obtidas por meio de um questionário e de uma entrevista e, após sistematização, realizada análise temática de conteúdo. Resultados: Participaram do estudo 51 ACS, sendo 84,3\% do sexo feminino; $64,7 \%$ na faixa etária de 25 a 40 anos; $86,2 \%$ compreendem que a principal via de transmissão do bacilo de Hansen ocorre pelas vias aéreas superiores, porém $13,8 \%$ reconhecem outras formas que não a correta; $96,1 \%$ reconhecem que os principais sinais e sintomas clínicos da hanseníase são manchas na pele e dormência; contudo, somente $88 \%$ conhecem a classificação operacional da doença. Da análise, emergiram duas categorias analíticas: Práticas na atenção aos casos de hanseníase e Qualificações recebidas sobre hanseníase.

Conclusão: Os ACS apresentam considerável conhecimento sobre a transmissão, sinais e sintomas e a classificação operacional da hanseníase e desenvolvem importantes práticas no território, no entanto, faz-se imperativo a continuidade de ações de educação permanente em saúde. Palavras-chave: Hanseníase. Agentes Comunitários de Saúde. Atenção Primária À Saúde.

\begin{abstract}
Objective: To describe the knowledge of community health agents (CHAs) about leprosy, its practices in the attention to the cases of its microarea and to identify the types of qualifications received about the disease.

Materials and Methods: Exploratory-descriptive study, under qualitative and quantitative approach. The subjects of the study were the CHAs who work in three territories of the Family Health Strategy (FHS) in the municipality of Sobral, Ceará, with the highest number of registered leprosy cases. The information was obtained through a questionnaire
\end{abstract}


and an interview and, after systematization, thematic content analysis was carried out.

Results: 51 CHAs participated in the study, of which $84.3 \%$ were female; $64.7 \%$ in the age group of 25 to 40 years; $86.2 \%$ understand that the main route of transmission of the Hansen bacillus occurs by the upper airways, but $13.8 \%$ recognize other forms than the correct one; $96.1 \%$ recognize that the main clinical signs and symptoms of leprosy are skin blemishes and numbness; however, only $88 \%$ are aware of the operational classification of the disease. From the analysis emerged two analytical categories: Practices in the attention to cases of leprosy and Qualifications received on leprosy.

Conclusions: CHAs present considerable knowledge about transmission, signs and symptoms, and the operational classification of leprosy and develop important practices in the territory, but it is necessary to continue the actions of permanent education in health.

Key-words: Leprosy. Community Health Agents. Primary HealTH CARE.

\section{INTRODUÇÃO}

A hanseníase é uma doença infecciosa crônica causada pelo Mycobacterium leprae, bacilo de alta infectividade e de baixa patogenicidade por infectar muitos, mas poucos manifestarem a doença. O homem é considerado a única fonte de infecção da hanseníase e a transmissão ocorre por meio das vias aéreas superiores de um sujeito doente na forma infectante multibacilar e sem tratamento. Sua sintomatologia é caracterizada por lesões na pele com hipoestesia ou anestesia, devido ao acometimento dos ramos sensitivos cutâneos. O diagnóstico é considerado essencialmente clínico e epidemiológico, sendo que, nos casos suspeitos, deve-se investigar a história, as condições de vida e realizar o exame dermato-neurológico para identificar lesões ou áreas de pele com alteração de sensibilidade e/ou comprometimento de nervos periféricos. ${ }^{1}$

Por se tratar de um problema de saúde pública, a hanseníase faz parte do rol de prioridades do Ministério da Saúde, com o desenvolvimento de ações vinculadas à vi- gilância em saúde, gestão, atenção integral, comunicação/educação e pesquisa por meio do Programa Nacional de Controle da Hanseníase $(\mathrm{PNCH}){ }^{1}$

De acordo com o Boletim Epidemiológico da Organização Mundial da Saúde (OMS), o Brasil é o país com o segundo maior número de casos notificados de hanseníase do mundo, depois da Índia. Em 2016, dos 27.356 casos novos das Américas, 92\% foram detectados no Brasil. ${ }^{2}$ Embora a situação epidemiológica da hanseníase no Brasil apresente tendência à estabilização dos coeficientes de detecção, as regiões Norte, Centro-Oeste e Nordeste mantêm altos índices. ${ }^{3}$ No Estado do Ceará a taxa de detecção de casos novos no ano de 2016 foi de 18,9/100.000 habitantes, parâmetro "muito alto" de endemicidade. ${ }^{4-5}$

Entre os 184 municípios do Estado do Ceará, cinco são prioritários para o $\mathrm{PNCH}$ do Ministério da Saúde, incluindo-se Sobral, cenário deste estudo. Sobral tem uma população de 201.756 habitantes 2014 e, nesse mesmo ano, diagnosticou 86 casos de hanseníase, com uma taxa de detecção de 
43,1 casos/100.000 habitantes, sendo classificado como muito alto por possuir um coeficiente de detecção entre 40,00/100.000 habitantes, segundo parâmetros do Ministério da Saúde. 5

O município de Sobral é referência para a Macrorregião da Saúde do Estado do Ceará, abrangendo 55 municípios, com atendimento a mais de 1.500 .000 usuários do Sistema Único de Saúde (SUS). O Sistema Municipal da Saúde de Sobral está organizado por uma rede de serviços próprios, conveniados e contratados. A Atenção Primária à Saúde (APS) está organizada a partir da Estratégia Saúde da Família (ESF), com 36 Centros de Saúde da Família (CSF) distribuídos entre Sede e distritos, com um total de 63 equipes, com 410 Agentes Comunitários de Saúde (ACS) territorializados em suas microáreas.

Quanto ao controle da hanseníase em âmbito municipal, faz-se necessária a atuação efetiva das equipes da ESF, em especial dos ACS, uma vez que estes atuam em contato direto e contínuo com a população; por, necessariamente, residirem na mesma área que atuam, são o elo entre a equipe e a comunidade, o que os faz vivenciarem o cotidiano da comunidade, com mais intensidade do que os demais trabalhadores da saúde ${ }^{7}$ e facilitação na busca ativa de sintomáticos dermatológicos. Como um trabalhador exclusivo do SUS, o ACS foi institucionalizado por meio do Programa Agente Comunitário de Saúde (PACS), criado em 1991 pelo Ministério da Saúde. Com a implantação do Programa Saúde da Família (PSF) em 1994, este ganhou destaque na APS, sendo considerado um elo importante para a identificação das necessidades de saúde das famílias e comunidades. $^{8}$
Quanto à atuação do ACS no controle da hanseníase, suas atribuições foram normatizadas no Guia de Controle da Hanseníase. ${ }^{9}$ Tais atribuições também estão estabelecidas no Artigo $3^{\circ}$. da Lei $n^{\circ}$. 11.350, de 5 outubro de 2006, que traz as atividades de prevenção de doenças e promoção da saúde, mediante ações nos lares ou comunidades, individuais ou coletivas, desenvolvidas em conformidade com as diretrizes do SUS. ${ }^{10}$

Considerando a relevância do papel do ACS para as ações de vigilância e controle da hanseníase em um município com muito alto parâmetro endêmico, o estudo objetiva descrever o conhecimento dos ACS sobre hanseníase, suas práticas na atenção aos casos nas microáreas e identificar as qualificações recebidas acerca da doença.

\section{Métodos}

Trata-se de um estudo exploratório-descritivo, sob abordagem quantitativa e qualitativa, realizado com 51 ACS no período de outubro de 2014 a setembro de 2015, no município de Sobral, Ceará. O estudo foi desenvolvido nos territórios da ESF dos bairros Terrenos Novos, Sumaré e Sinhá Saboia da Sede de Sobral. Estes foram escolhidos por apresentarem os maiores coeficientes de detecção de casos novos de hanseníase no período de 2010 a 2014. As taxas de detecção nesses bairros, no ano de 2014, foram: 81 casos/100.000 habitantes em Terrenos Novos, 71,4 casos/100.000 habitantes no Sumaré e 58,1 casos/100.000 no Sinhá Sabóia, ${ }^{11}$ sendo esses territórios hiperendêmicos.

O critério de inclusão dos participantes foi o de estarem inseridos na ESF do terri- 
tório há um ano ou mais. De um total de 58 ACS identificados, 51 estavam aptos a participar da pesquisa. O contato com os ACS foi estabelecido nos Centros de Saúde da Família (CSF) a que estavam vinculados, sendo todos esclarecidos sobre os objetivos do estudo, a garantia de sigilo de suas identidades e o direito de desistir de participar da pesquisa a qualquer momento. Foi apresentado a cada participante um Termo de Consentimento Livre e Esclarecido, que foi assinado por todos. Como forma de resguardar o sigilo e preservar a identidade dos ACS, estes foram denominados pela sigla "ACS" seguido de um numeral arábico, como sendo: ACS 1, ACS 2, ACS 3 e assim sucessivamente.

Para a obtenção das informações, utilizaram-se dois instrumentos: um questionário com perguntas objetivas para levantar dados de identificação dos ACS, do seu conhecimento em relação aos sinais e sintomas, do modo de transmissão e da classificação da hanseníase; e o outro instrumento foi um roteiro de entrevista, com perguntas subjetivas para investigar as práticas na atenção aos casos de sua área, bem como, identificar os tipos de qualificações recebidas acerca da hanseníase.

As questões subjetivas da pesquisa foram respondidas por um número menor de ACS por se considerar que, no todo, as características dessas respostas poderiam se tornar repetitivas em sua análise. Na seleção dos respondentes dessa parte da pesquisa, utilizou-se a técnica de escolha de amostragem não probabilística proporcional, tendo como quota a proporção de uma entrevista a cada seis participantes abordados; com isso, chegou-se a um número de oito participantes para responder a essas questões. A ideia de amostragem por proporção sugere que se os participantes são representativos em termos de características, eles também poderão ser representativos em termos da informação procurada pela pesquisa. ${ }^{12}$ As entrevistas foram gravadas em um gravador e, posteriormente, transcritas de forma fidedigna e integral.

Após a transcrição das entrevistas dos ACS, foi efetuada uma leitura com vistas a sistematizar os principais sentidos destas em relação aos objetivos do estudo, além de categorizá-los de acordo com o referencial de Minayo, ${ }^{13} \mathrm{com}$ a análise a partir de quatro etapas: pré-análise, exploração do material, tratamento dos resultados obtidos e interpretação. As informações quantitativas obtidas no questionário foram sistematizadas e tratadas por meio da planilha eletrônica de dados da Microsoft Excel ${ }^{\circ}$, Versão 2010. Em seguida, os resultados foram analisados a partir da estatística descritiva simples e apresentados em forma de gráficos e tabelas, dialogando com a literatura pertinente ao tema.

O estudo foi apreciado e aprovado pela Comissão Científica da Secretaria da Saúde de Sobral, responsável pela análise das pesquisas realizadas no Sistema Municipal da Saúde de Sobral e, em seguida, pelo Comitê de Ética em Pesquisa (CEP) da Universidade Estadual Vale do Acaraú (UVA), recebendo parecer favorável $\mathrm{n}^{\circ} .1 .061 .742$ e CAAE $\mathrm{n}^{\circ}$. 42510815.5.0000.5053. Por se tratar de uma investigação no âmbito da saúde que envolveu a participação de seres humanos, o estudo obedeceu aos princípios da Resolução nº. 466, de 12 de dezembro de 2012, do Conselho Nacional de Saúde sobre pesquisa envolvendo seres humanos. ${ }^{14}$ 


\section{RESUlTADOS E DISCUSSÃo}

A Tabela 1 apresenta os dados sociodemográficos dos ACS. Os dados acerca do perfil dos ACS foram inseridos no estudo para se ter uma maior clareza durante a análise dos resultados de quem são, sua formação e tempo de trabalho no território, o que contribui com o reconhecimento deste e dos determinantes, além de apresentar certa maturidade profissional e consolidação do vínculo com a comunidade.

Tabela 1 - Perfil sociodemográfico dos agentes comunitários de saúde. Sobral Ceará, Brasil, 2015.

\begin{tabular}{l|r|r}
\hline \multicolumn{1}{c|}{ Variáveis } & N & \% \\
\hline Sexo & & \\
Feminino & 43 & 84,3 \\
Masculino & 8 & 15,7 \\
$\quad$ Total & 51 & 100,0 \\
Faixa Etária (anos) & & \\
25-30 & 10 & 19,6 \\
$31-40$ & 23 & 45,1 \\
$41-50$ & 13 & 25,5 \\
$>50$ & 5 & 9,8 \\
$\quad$ Total & 51 & 100,0 \\
Escolaridade & 1 & 1,9 \\
Ensino Médio Incompleto & 39 & 76,5 \\
Ensino Médio Completo & 8 & 15,7 \\
Universitário Incompleto & 3 & 5,9 \\
Universitário Completo & 51 & 100,0 \\
$\quad$ Total & & \\
Tempo de trabalho no & 19 & 37,2 \\
território (anos) & 27 & 53,0 \\
1-9 & 5 & 9,8 \\
$10-19$ & 51 & $\mathbf{1 0 0 , 0}$ \\
$\geq 20$ &
\end{tabular}

A Tabela 1 supracitada revela a predominância do sexo feminino como força de trabalho, nos serviços de saúde, esse fato não é novidade, pois estudos semelhantes demonstraram que, no Brasil, o perfil da força de trabalho nos serviços de saúde e na ESF tem predominância feminina em torno de $80 \%$ dos trabalhadores. ${ }^{15}$

Os ACS, predominantemente encontram-se na faixa etária entre 31 e 40 anos, fase importante da vida produtiva e de maturidade profissional. A maioria possui o ensino médio completo. A lei que cria a profissão do ACS estabelece o ensino médio como requisito de escolaridade mínima para o exercício da profissão e a obrigatoriedade de passar por uma qualificação básica de 400 horas. Para efetivar essa qualificação, o Ministério da Saúde propôs um itinerário de formação do ACS até que atinja o nível técnico (1.200 horas), o que exige, além da qualificação profissional, o ensino médio. ${ }^{16}$ À medida que o ACS atua como elemento integrador entre a comunidade e o serviço de saúde, torna-se fundamental refletir sobre o seu papel na equipe e na mudança do modelo de atenção, ${ }^{17}$ sendo a qualificação profissional um importante fator para o fortalecimento das ações.

Os ACS apontaram como principal via de transmissão do bacilo da hanseníase as vias aéreas superiores; no entanto, referiram também que o contato com a pele pode transmitir a doença. Essa ideia pode estar relacionada ao fato de a hanseníase ter como uma de suas características clínicas principais as alterações dermatológicas. Em estudo semelhante observou-se que a hanseníase é concebida no imaginário popular como uma doença que pode ser transmitida por um simples contato de pele e que se transmite enquanto a lesão estiver presente. ${ }^{18}$ 
Tabela 2 - Conhecimento dos agentes comunitários de saúde sobre transmissão, sinais e sintomas e classificação operacional da hanseníase. Sobral - Ceará, Brasil, 2015.

\begin{tabular}{l|r|r}
\hline \multicolumn{1}{c|}{ Variáveis } & N & $\%$ \\
\hline Modo de Transmissão & 44 & 86,2 \\
Vias áreas superiores & 6 & 11,8 \\
Pele & 1 & 2,0 \\
Compartilhamento de objetos & 51 & 100,0 \\
Total & & \\
Classificação Operacional & 2 & 4,0 \\
Paucibacilar e Lepromatosa & 1 & 2,0 \\
Lepromatosa e Verrugosa & 3 & 6,0 \\
Verrugosa e Multibacilar & 45 & 88,0 \\
Multibacilar e Paucibacilar & 51 & 100,0 \\
Total & & \\
Sinais e Sintomas* & 3 & 5,9 \\
Manchas escuras na pele & 49 & 96,1 \\
Manchas na pele com dormência & 33 & 64,7 \\
Manchas esbranquiçadas ou & & \\
avermelhadas & 1 & 2,0 \\
Prurido (coceira) & 27 & 52,9 \\
Dor ou sensibilidade nos nervos & & \\
\hline & &
\end{tabular}

${ }^{\star} \mathrm{O}$ n diverge ao do estudo por conta dos sujeitos terem apontado mais de uma opção de sinais e sintomas.

Contudo, a ausência de conhecimento sobre a doença favorece a transmissão, uma vez que ocorre por meio da eliminação do bacilo para o meio exterior através da tosse, espirro ou fala de um doente que não está em tratamento, entre sujeitos com contato direto, frequente, íntimo e prolongado, e não pelo simples encontro social com o sujeito doente. Entretanto, é importante destacar que nem todos os expostos ao bacilo desenvolvem a doença. ${ }^{8}$

$\mathrm{Na}$ Tabela 2 observa-se que os sujeitos do estudo demonstraram saber reconhecer os sinais e sintomas clínicos da hansenía- se, como manchas na pele com dormência, manchas esbranquiçadas ou avermelhadas e dor ou sensibilidade nos nervos, conhecimento relevante para a busca ativa de sujeitos sintomático-dermatológicos e para se realizar o diagnóstico precoce da hanseníase. Tais resultados corroboram com o papel do ACS no controle da hanseníase com a suspeita precoce dos casos existentes na comunidade e o encaminhamento destes para confirmação diagnóstica e realização do tratamento no CSF. ${ }^{15}$

O papel do ACS é essencial para a interrupção da cadeia de transmissão à medida, principalmente, que estes reconhecem os sintomas da doença, encaminham corretamente os casos suspeitos para investigação do diagnóstico e colaboram para o início precoce do tratamento. Evitam-se, assim, as fontes de contaminação e reduzem-se as sequelas, já que a hanseníase é uma das principais causadoras de incapacidades entre as doenças infectocontagiosas. ${ }^{19}$

Uma das estratégias de controle da hanseníase consiste na divulgação intensa de suas manifestações clínicas junto à comunidade. Para isso, os profissionais da saúde devem estar qualificados para reconhecer tais sinais e sintomas, inclusive o ACS, visto que é ele o profissional responsável por formar o elo entre a população adscrita e a equipe da ESF. Uma das suas atribuições é estar em contato permanente com as famílias desenvolvendo atividades educativas, visando a promoção da saúde. ${ }^{20}$

A Tabela 2 apresenta o conhecimento dos ACS em relação à classificação operacional da hanseníase. Por meio desses achados, verifica-se que a maior parte dos ACS conhecem a classificação operacional 
da doença em Multibacilar (MB) e Paucibacilar (PB). Classificar os sujeitos doentes em $\mathrm{MB}$ e $\mathrm{PB}$ é relevante, pois o tratamento difere para os dois grupos. O Ministério da Saúde classifica a hanseníase para fins operacionais de tratamento quimioterápico em $\mathrm{PB}$ os casos com até cinco lesões de pele, que corresponde às formas Tuberculoide e Indeterminada; e MB os casos com mais de cinco lesões de pele, nas formas Virchowiana e Dimorfa. ${ }^{5}$

Ainda, a importância de se conhecer a classificação da hanseníase está relacionada ao acompanhamento do tratamento, às manifestações e compreender as reações, atividade que está inserida no cotidiano do ACS, e que contribuirá para um acompanhamento mais específico do andamento e tratamento dos casos.

\section{Práticas na atenção aos CASOS DE HANSENÍASE}

A partir das entrevistas realizadas com oito ACS, surgiram duas categorias temáticas para a análise do objeto do estudo: Práticas na atenção aos casos de hanseníase e Qualificações recebidas sobre hanseníase.

A diversidade e a heterogeneidade das atividades desenvolvidas pelo ACS destacam-se como ponto central das orientações, especialmente a respeito da prevenção de doenças e do acompanhamento de tratamento. O profissional da saúde, ao se aproximar da realidade social e cultural do doente, possibilita o desenvolvimento de um trabalho conjunto, dialogando sobre as propostas do tratamento, as orientações em relação ao autocuidado e as possíveis adaptações necessárias a fim de minimizar o impacto da doença na vida dos sujeitos. ${ }^{15}$
Uma das tecnologias utilizadas pelos ACS durante o seu labor no território da ESF é a visita ao lar, que é carregada de subjetividade para construção e manutenção do vínculo com as famílias e comunidades. As falas a seguir retratam o trabalho do ACS durante as visitas aos sujeitos com hanseníase:

\begin{abstract}
Durante a visita na casa do paciente vejo se a medicação está fazendo algum efeito para ele, se está se sentindo bem. Tem paciente que não se dá bem, dá uma reação (ACS 1).
\end{abstract}

No momento que eles começam o tratamento, então a gente já visita e conversa com a familia, que a família precisa ir na unidade. Então, marca o dia com a enfermeira e encaminha pela familia as pessoas que têm mais contato com ele para serem examinados (ACS 3).

Os ACS relataram durante as entrevistas que o instrumento de trabalho mais utilizado no cuidado com a saúde da população são as visitas, momento que é possível orientar as famílias sobre a prevenção de riscos, agravos e doenças e realizar a promoção da saúde individual e de seus entes. A visita é uma ferramenta fundamental para a efetividade dos serviços na ESF, pois permite ao profissional conhecer a realidade de vida de sua clientela, condições de habitação, relações familiares, entre outros. Promove, ainda, o planejamento da atenção, da assistência e do cuidado por enunciar o reconhecimento dos recursos que a família dispõe. ${ }^{8}$

A visita éuma ferramenta imprescindível para o cuidado no próprio lar, uma vez que este representa o lócus essencial para o desenvolvimento de um plano de cuidados efetivo, integral, responsável, ético e humanizado. Durante a visita ao lar, é possível reconhecer as condições de vida e o proces- 
so saúde-doença-cuidado das famílias, dos sujeitos e das comunidades, bem como intervir quando necessário. Embora a terminologia nacionalmente conhecida seja visita domiciliar, o termo lar propicia maior acolhimento, construção de vínculo e desenvolvimento do processo de cuidar holístico e humanizado. ${ }^{21}$

A visita ao lar, seja para educação em saúde, busca ativa, encaminhamento, detecção, acompanhamento ou exame de contatos de sujeitos com hanseníase, é uma importante prática sanitária. A visita fortalece a adesão ao tratamento instituído, haja vista que a hanseníase exige uma terapêutica e um acompanhamento de longo prazo, além do fato da medicação usada poder levar a diversas reações: ${ }^{22}$

Eu faço orientações na casa do paciente em relação aos cuidados né, quanto calçados, e se dependendo também do trabalho dele, tem que ter cuidado com tudo isso, não se expor ao sol e usar o protetor solar (ACS 2).

E paras as pessoas que moram na casa eu peço para tomar a vacina na unidade... (ACS 5).

No tocante às orientações dos ACS aos sujeitos com diagnóstico de hanseníase, foram identificados relatos reforçando a importância do tratamento correto e do autocuidado. Ressalta-se, também, o acompanhamento/avaliação dos contatos e a vacinação BCG. Para os casos de hanseníase, é recomendada a administração de BCG em contatos domiciliares, independentemente da idade, exceto naqueles com HIV. ${ }^{23}$

Outra prática relevante no cuidado ao sujeito com hanseníase desenvolvida pelo ACS são as orientações e os diálogos com os sujeitos sobre o preconceito e o estigma da doença:

\begin{abstract}
Procuro falar sobre a questão do estigma, do preconceito que, muitas das vezes, eles mesmos quando estão fazendo o tratamento tendem querer se excluir da família, do grupo, do trabalho. Explico que a partir que faz o tratamento ele não transmite mais a doença (ACS 6).
\end{abstract}

Oriento assim na questão de ele ver como ele passa para as pessoas, porque existe preconceito mesmo que a gente não diga que não tem, mas tem. Então a gente conversa sobre isso (ACS7).

A hanseníase é vista como uma doença a ser temida devido às deformidades físicas apresentadas pelos sujeitos não tratados, gerando estigmas, preconceito e superstições. Em se tratando de hanseníase, o preconceito se refere à desqualificação e à marginalização social, consequência das deformidades físicas. ${ }^{24}$

Dessa forma, o ACS tem o papel de orientar e divulgar informações adequadas sobre a hanseníase para desmistificar a imagem negativa associada à doença desde tempos remotos da História. É necessário repensar as práticas frente ao doente e aos seus familiares, rompendo com a ideia que se perpetua de que essa doença é algo danoso para aqueles que conviverem com ela, ao passo que, na atualidade, pode ser vista como algo muito simples, especialmente quando tratada corretamente e em tempo hábil. ${ }^{19}$

\section{QUALIFICAÇÕES RECEBIDAS SOBRE HANSENÍASE}

A capacitação dos profissionais que realizam assistência aos sujeitos e familiares 
com hanseníase constitui um dos resultados esperados com o desenvolvimento do $\mathrm{PNCH}$, estabelecido pelo Ministério da Saúde, a fim de contribuir com o controle da doença nos municípios. ${ }^{5}$ As falas evidenciam a participação dos ACS em alguns momentos de qualificação:

Teve uma capacitação fazendo um comparativo de quantos casos têm em cada bairro, os tratamentos e a mobilização quanto a mancha que acontece nas unidades (ACS 4).

Eu me lembro de um. Esse momento foi mais para clarear o que a gente realmente já conhece né, foi mais assim para a gente ter uma noção de quanto está em todo o município (ACS 5).

Já fui para a curso que falou sobre a hanseníase, principalmente dos sintomas e como transmite (ACS 8).

Observa-se que as qualificações se dão por meio de capacitações ou de atualizações quase sempre pontuais, o que pode se refletir como uma fragilidade por não proporcionarem uma abordagem contínua dos problemas encontrados na prática profissional, o que pode limitar transformações das práticas, a melhoria da busca ativa e a efetividade do tratamento. A fim de esclarecimento, cabe destacar que não há uma ideia que nega a importância de capacitações pontuais, entretanto, acredita-se que, de forma isolada, não se desencadeiam resultados práticos no cuidado às famílias, por isso necessitando ocorrer na dimensão em que se compreende a realidade e as necessidades dos trabalhadores das equipes e da gestão do trabalho. ${ }^{25}$

Assim, o controle da hanseníase depende, também, de informações adequadas que esses e outros profissionais repassam à população, além do compromisso que buscam resolubilidade no tratamento e reabilitação dos casos. Desse modo, cabe aqui o debate da necessidade de um processo constante de avaliação sobre a qualificação dos ACS compatível com a complexidade dos problemas de saúde da população e de sua microárea. Nesse contexto, o processo de capacitação e de qualificação dos ACS é fundamental para que eles adquiram competências e as implementem com eficiência na sua rotina de trabalho para melhorar a qualidade da assistência prestada à comunidade.

\section{CONSIDERAÇões FINAIS}

Diante dos depoimentos dos participantes deste estudo, foi possível perceber que os ACS apresentam considerável conhecimento sobre a transmissão, sinais e sintomas e a classificação operacional da hanseníase e desenvolvem importantes práticas no território como: a visita ao lar aos sujeitos com hanseníase, orientações quanto à importância do tratamento correto e do autocuidado. Ressalta-se, também, o acompanhamento dos contatos, a vacinação BCG, o diálogo com os doentes a respeito do preconceito, do estigma da doença e da participação pontual em momentos de capacitações.

Considerando que o ACS tem participação na meta de eliminação da hanseníase como um problema de saúde pública no país, esse profissional deve ser treinado e receber atualizações na área de vigilância e controle da hanseníase de forma continuada, pois essas atividades, quando se tornam apenas pontuais, podem enfraquecer a investida de 
formação de recursos humanos como estratégia para o controle e a eliminação da hanseníase como problema de saúde pública.

Compreendemos que, em virtude de o estudo ter sido realizado apenas nas áreas com alta carga da doença, ainda mais em se tratando de um município endêmico, os casos de hanseníase podem estar deixando de serem detectados porque os profissionais não estão bem qualificados para identificar casos suspeitos da doença.

O conhecimento produzido por este estudo sugere a elaboração de estratégias educacionais de disseminação do conhecimento sobre a doença, de modo que sirva de subsídio na prevenção e detecção precoce de casos de hanseníase, pautadas na relevância que o ACS possui enquanto membro da equipe de saúde e na sua proximidade com a população.

\section{REFERÊNCIAS}

1. Brasil. Ministério da Saúde. Secretaria de Atenção à Saúde. Departamento de Atenção Básica. Vigilância em Saúde: Dengue, Esquistossomose, Hanseníase, Malária, Tracoma e Tuberculose, 2. ed. rev. [Internet]. Brasília: Ministério da Saúde; 2008, 197 p.: il. - (Série A. Normas e Manuais Técnicos) (Cadernos de Atenção Básica, n. 21) [acesso em 2017 jul. 31]. Disponível em: http:// bvsms.saude.gov.br/bvs/publicacoes/cab_n21_vigilancia_saude_2ed_p1.pdf.

2. World Health Organization. Global leprosy update, 2016: accelerating reduction of disease burden. Weekly epidemiological record. [Internet] 2017 [acesso em 2017 out. 25]. Sep.; 35, 2017, (92), 501-520. Disponível em: http://apps.who.int/iris/bitstream/10665/258841/1/WER9235. pdf?ua $=1$

3. Silva MCD, Paz EPA. Educação em saúde no programa de controle da hanseníase: a vivência da equipe multiprofissional. Esc. Anna Nery [Internet], 2010 [acesso 2017 out. 25] jun. 14 (2): 223-229. Disponível em: http://www.scielo.br/scielo.php?script=sci_arttext\&pid=S1414-81452010000200003\&lng=en. http://dx.doi.org/10.1590/S1414-81452010000200003.

4. Secretaria da Saúde do Estado do Ceará. Coordenadoria de Promoção e Proteção à Saúde. Núcleo de Vigilância Epidemiológica. Informe Epidemiológico Hanseníase [Internet]. Fortaleza: Secretaria da Saúde do Estado do Ceará; 2016 [acesso em 2017 set. 30]. Disponível em: http:// www.saude.ce.gov.br/index.php/boletins?download=3327\%3Aboletim-epidemiologico-hanseniase--5-de-setembro-de-2017.

5. Brasil. Ministério da Saúde. Secretaria de Vigilância em Saúde. Departamento de Vigilância das Doenças Transmissíveis. Diretrizes para vigilância, atenção e eliminação da Hanseníase como problema de saúde pública: manual técnico-operacional [Internet]. Brasília: Ministério da Saúde, 2016, 58 p. [acesso em 2017 jul. 31]. Disponível em: http://portalarquivos.saude.gov. br/images/pdf/2016/fevereiro/04/diretrizes-eliminacao-hanseniase-4fev16-web.pdf.

6. Brasil. Instituto Brasileiro de Geografia e Estatística (IBGE). Censo Demográfico [Internet]. Ceará: IBGE; 2015 [acesso em 2015 dez. 15]. Disponível em: http://cod.ibge.gov.br/234TM.

7. Nogueira RP, Silva FBS, Ramos ZVO. A vinculação institucional de um trabalhador sui generis: o agente comunitário de saúde [Internet]. Rio de Janeiro: Cadernos do IPEA; 2000, p. 2 [acesso em 2017 jul. 31]. Disponível em: http://www.en.ipea.gov.br/agencia/images/stories/ PDFs/TDs/td_0735.pdf.

8. Furlan PG. O agente comunitário de saúde e a prática na atenção básica: alguns apontamentos. In: Campos GWS (Org.). Manual de práticas de atenção básica: saúde ampliada e compartilhada. São Paulo: Aderaldo \& Rothschild; 2008. 
9. Brasil. Ministério da Saúde Secretaria de Políticas de Saúde. Departamento de Atenção Básica. Guia para o Controle da hanseníase [Internet]. Brasília: Ministério da Saúde, 2002, p.:il. (Série A. Normas e Manuais Técnicos; n. 111) [acesso em 2017 jul. 31]. Disponível em: http:// bvsms.saude.gov.br/bvs/publicacoes/guia_de_hanseniase.pdf.

10. Brasil. Presidência da República, Subchefia para Assuntos Jurídicos. Lei No. 11.350, de 5 de outubro de 2006. Dispõe sobre o aproveitamento de pessoal amparado pelo parágrafo único do art. $2^{\circ}$. da Emenda Constitucional $n^{\circ} .51$, de 14 de fevereiro de 2006, e dá outras providências [Internet]. Brasília, DF; 2006 [acesso em 2015 out. 29]. Disponível em: http: //www.unipac.br/ site/bb/guias/Normas\%20Vancouver\%20-\%202017.pdf

11. Secretaria Municipal de Saúde de Sobral. Sistema de Informação de Agravos de Notificação. SINAN Local. Sobral: Secretaria Municipal de Saúde de Sobral; 2014.

12. Curwin J., Slater R. Quantitative methods for business decisions, 3. ed. Chapman \& Hall; 1991.

13. Minayo MCS. O desafio do conhecimento: pesquisa qualitativa em saúde, 11. ed. São Paulo: Hucitec; 2014.

14. Brasil. Resolução № 466 do Conselho Nacional de Saúde, de 12 de dezembro de 2012. Aprova as diretrizes e normas regulamentadoras de pesquisas envolvendo seres humanos [Internet]. Diário Oficial da União, 12 dez. 2012 [acesso em 2015 out. 29]. Disponível em: http://conselho. saude.gov.br/resolucoes/2012/Reso466.pdf

15. Pereira IDF, Lopes MR, Nogueira ML, Ruela HCG. Princípios pedagógicos e relações entre teoria e prática na formação de agentes comunitários de saúde. Trab. Educ. Saúde [Internet], 2016 [acesso em 2017 out. 25]; 14 (2): 377-397. Disponível em: http://www.scielo.br/scielo.php?script=sci_arttext\&pid=S1981-77462016000200377\&lng=en\&nrm=iso. http://dx.doi. org/10.1590/1981-7746-sol00010

16. Brasil. Ministério da Saúde. Ministério da Educação. Referencial curricular para curso técnico de agente comunitário de saúde: área profissional saúde [Internet]. Brasília: Ministério da Saúde; 2004, 64 p.: il. color. - (Série A. Normas e Manuais Técnicos) [acesso em 2017 jul. 31]. Disponível em: http://bvsms.saude.gov.br/bvs/publicacoes/referencial_Curricular_ACS.pdf.

17. Shon DA. Educando o Profissional Reflexivo: um novo design para o ensino aprendizagem. Porto Alegre: Artmed; 2000.

18. Gomes FC, Oliveira TC, Araújo JER, Félix LG, Araújo KMFA. Conhecimento do usuário da atenção primária à saúde acerca da hanseníase. Rev Enferm UFPE [Internet], 2014 [acesso em 2017 jul. 31]; 8 (2): 3669-76. Disponível em: https://periodicos.ufpe.br/revistas/revistaenfermagem/article/view/10108/10580.

19. Sales JCS, Luz VLES, Branco FMF, Araújo MFF, Castro SHTS, Silva TCA. O significado da hanseníase para o agente comunitário de saúde. R. Interd. 2013; 6 (1): 17-24.

20. Cruz PSC, Oda JY. Atuação dos agentes comunitários de saúde no programa de controle da hanseníase em um município do Noroeste do Paraná. Arquivos de Ciências da Saúde da UNIPAR [Internet], 2009 [acesso em 2017 jul. 31]; 13 (3): 217-222. Disponível em: http://revistas.unipar. br/index.php/saude/article/view/3202/2240

21. Ximenes Neto FRG, Chaves ME, Ponte MAC, Cunha ICKO. Trabalho do enfermeiro da Estratégia Saúde da Família na visita ao lar da puérpera e recém-nascido. Revista da Sociedade Brasileira de Enfermeiros Pediatras [Internet], 2012 [acesso em 2017 jul. 31]; 12 (1): 27-36. Disponível em: http://www.sobep.org.br/revista/component/zine/article/150-trabalho-do-enfermeiro-da-estratgia-sade-da-famlia-na-visita-ao-lar-da-purpera-e-recm-nascido.html

22. Andrade CG, Costa ICP, Freire MEM, Santos KFO, Gouveia EML, Claudino HG. Hanseníase: Compreensão de Agentes Comunitários de Saúde. Braz. J. Surg. Clin. Res. [Internet], 2011 [acesso em 2017 jul. 31];15 (1): 17-24. Disponível em: http://periodicos.ufpb.br/index.php/rbcs/ article/view/9914/5810 
23. Brasil. Ministério da Saúde. Secretaria de Atenção à Saúde. Departamento de Atenção Básica. Vigilância em Saúde: Dengue, Esquistossomose, Hanseníase, Malária, Tracoma e Tuberculose, 2. ed. rev. [Internet]. Brasília: Ministério da Saúde; 2008, 195 p.: il. - (Série A. Normas e Manuais Técnicos) (Cadernos de Atenção Básica, n. 21) [acesso em 2017 jul. 31]. Disponível em: http:// bvsms.saude.gov.br/bvs/publicacoes/cab_n21_vigilancia_saude_2ed_p1.pdf

24. Silva Junior FJG, Ferreira RD, Araújo OD, Camêlo SMA, Nery IS. Assistência de enfermagem ao portador de Hanseníase: abordagem transcultural. Rev. bras. enferm. [Internet], 2008 nov. [acesso 2017 out. 25]; 61 (spl): 713-717. Disponível em: http://www.scielo.br/scielo. php?script=sci_arttext\&pid=S0034-71672008000700010\&lng=en. http://dx.doi.org/10.1590/ S0034-71672008000700010.

25. Donaduzzi DSS. A educação para o trabalho na perspectiva dos Agentes comunitários de saúde [dissertação] [Internet]. Santa Maria: Universidade Federal de Santa Maria; 2012 [acesso em 2017 out. 25]. Disponível em: http://coral.ufsm.br/ppgenf/images/Mestrado/Dissertacoes/2012_2013/Dissertacao_Daiany_Saldanha_da_Silveira_Donaduzzi.pdf.

\section{DADOS DO(S) AUTOR(ES)}

\section{CAMILA MARTINS DE OLIVEIRA}

Enfermeira pela Universidade Estadual Vale do Acaraú, Sobral/ CE - Brasil. martinscamila75@ gmail.com

\section{MARIA SOCORRO CARNEIRO LINHARES}

Enfermeira Doutoranda em Saúde Coletiva da Universidade Federal do Ceará (UFC), Docente do curso de Graduação em Enfermagem da Universidade Estadual Vale do Acaraú e do Mestrado em Saúde da Família pela RENASF/UVA/FIOCRUZ, Sobral, Ceará, Brasil. socorrocarneiro1@gmail. com

\section{FRANCISCO ROSEMIRO GUIMARÃES XIMENES NETO}

Enfermeiro Doutor em Ciências pela Escola Paulistana de Enfermagem/ Universidade Federal de São Paulo (Unifesp). Docente do Curso de Graduação em Enfermagem da Universidade Estadual Vale do Acaraú e do Mestrado em Saúde da Família pela RENASF/UVA/FIOCRUZ, Universidade Estadual Vale Do Acaraú, Sobral/ CE - Brasil. rosemironeto@gmail.com

\section{IRISDALVA MARIA VIEIRA PESSOA MENDES}

Especialista em Dermatologia Sanitária. Médica do Ambulatório de Referência para Secretaria de Saúde de Sobral/ CE - Brasil. irisdalvapessoa@yahoo.com.br

\section{LÍGIA REGINA FRANCO SANSIGOLO KERR}

Médica Pós-Doutorada em Epidemiologia na Harvard School Of Public Health e Pós-Doutorada em Epidemiologia na University Of California San Francisco.

Docente Da Universidade Federal do Ceará (UFC), Fortaleza/ CE - Brasil. ligiakerr@gmail.com

Submetido em: 10/03/2017

Aceito em: 30/01/2018 